\title{
Interference Mitigation by Adaptive Analog Spatial Filtering for MIMO Receivers
}

\author{
Masoud Abbasi Alaei, Student Member, IEEE, Sajad Golabighezelahmad, Student Member, IEEE, \\ Pieter-Tjerk de Boer, Frank E. van Vliet, Senior Member, IEEE, Eric A. M. Klumperink, Fellow, IEEE, and \\ André B. J. Kokkeler, Member, IEEE
}

\begin{abstract}
Exposed to strong co-channel/adjacent channel interference, digital Multiple-Input Multiple-Output (MIMO) receivers require high dynamic range Analog-to-Digital Converters (ADCs). Hybrid analog-digital beamforming featuring spatial filtering before the ADCs can adaptively mitigate interference in both the analog and digital domains; hence, relaxing the required dynamic ranges of the ADCs. This paper demonstrates the effectiveness of hybrid beamforming in this mitigation utilizing adaptive Minimum Mean Square Error (MMSE) and Error Vector Magnitude (EVM) as an optimization criterion. Extensive EVM measurements are carried out with a conductive set-up using a 4-element $22 \mathrm{~nm}$ FD-SOI CMOS prototype MIMO receiver chip to verify the performance of the adaptive MMSE algorithm. Over-the-Air (OTA) measurements with a linear 4element dipole antenna array with half-wavelength spacing in the 2.4 GHz ISM-band quantify the improvement for real world scenarios, e.g., having a multipath propagation channel and mutual coupling between the antenna array elements. OTA results show that a rejection of $22.5 \mathrm{~dB}$ and $24.5 \mathrm{~dB}$ can be achieved on average in an in-door laboratory environment utilizing Vector Modulator (VM) constellations with 16 and 64 points, respectively.
\end{abstract}

Index Terms-Adaptive spatial filtering, Hybrid beamforming, Multiple-Input Multiple-Output (MIMO), Receiver, Vector Modulator, Interference rejection, Software-defined radio.

\section{INTRODUCTION}

D IGITAL MIMO systems have recently attracted increasing attention, because they allow for spatial multiplexing and diversity gains, improving link throughput and link reliability. As the spectrum becomes heavily utilized in sub-GHz bands, strong mutual interference can occur. Digital MIMO offers a solution for efficient use of scarce radio spectrum [1]. Although a digital MIMO receiver allows for spatial filtering in the digital domain, thereby reducing interference,

This work was supported by Dutch Research Council (NWO) through the TTW-Research Program MIRABEAM under Project 14689. (Masoud Abbasi Alaei and Sajad Golabighezelahmad are co-first authors.)

Masoud Abbasi Alaei and André B. J. Kokkeler are with the Radio Systems (RS) group, Faculty of Electrical Engineering, Mathematics, and Computer Science, University of Twente, 7522 NB Enschede, The Netherlands.

Sajad Golabighezelahmad and Eric A. M. Klumperink are with the IC Design Group, Faculty of Electrical Engineering, Mathematics, and Computer Science, University of Twente, 7500 AE Enschede, The Netherlands.

Pieter-Tjerk de Boer is with the Design and Analysis of Communication Systems (DACS) group, Faculty of Electrical Engineering, Mathematics, and Computer Science, University of Twente, 7522 NB Enschede, The Netherlands.

Frank E. van Vliet is with TNO, 2509 JG The Hague, The Netherlands, and also with the IC Design Group, Faculty of Electrical Engineering, Mathematics, and Computer Science, University of Twente, 7500 AE Enschede, The Netherlands. the RF front-ends and ADCs are still susceptible to strong in-band interferers. Therefore, the distortion at the nonlinear RF/Analog front-end and clipping effects at the ADCs can easily degrade performance (see Fig. 1a). Hence, strong cochannel/adjacent channel interference needs to be addressed.

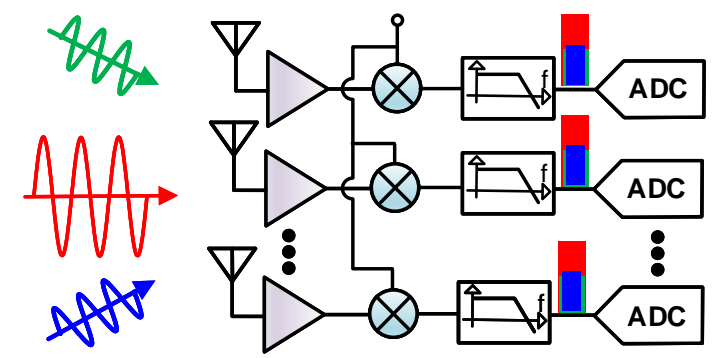

(a)

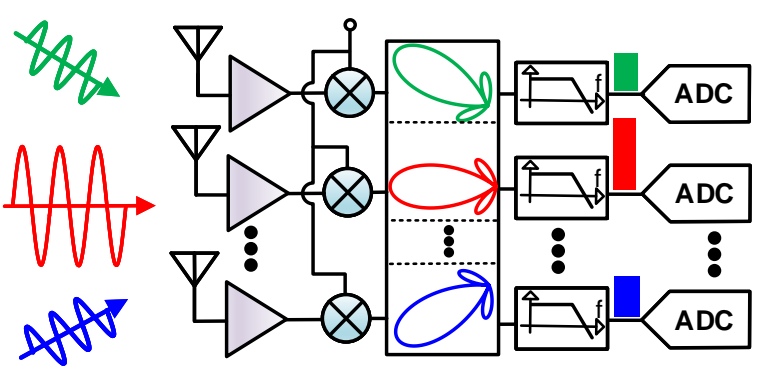

(b)

Fig. 1. (a) Digital MIMO receivers with separate receive chains are susceptible to a strong co-channel interferer; (b) Analog multi-beamforming can reduce this interference while still receiving signals from all directions.

To overcome the distortion caused by the nonlinear $\mathrm{RF} / \mathrm{analog}$ front-ends and high dynamic range requirements for the ADCs in digital MIMO receivers, hybrid beamforming MIMO receiver architectures have been proposed [2]-[4]. $\mathrm{RF} /$ analog beamforming before the ADCs can reduce interference by spatial filtering as shown in Fig. 1b, while identical receive chains without analog beamforming all get exposed to strong interference as in Fig. 1a. The focus of this work is to show the effectiveness of hybrid beamforming in interference mitigation. A few research papers partially address this, e.g., exploring the theoretical aspects of interference mitigation by hybrid beamforming MIMO receivers [5], [6], but they lack experimental results that show the effectiveness of spatial filtering in practice. Reference [5] merely uses simulations to show the reduction in the number of ADC bits and ADC power consumption by adaptive spatial suppression. In [6], a design technique was proposed for an optimal analog beamformer, 
minimizing the mean square error by online channel estimation, i.e., estimating the statistics of the wireless channel. Although interesting for our goal, reference [6] assumes that only phase shift control is available in the analog domain and it relies on simulation results only. A recent paper [7] uses a passive block called a Spatial Interference Mitigation Circuit (SMIC) placed before the RF front-end to create an spatial notch for MIMO receiver elements. References [2][4], [8]-[19] do demonstrate the design of interference robust techniques using CMOS integrated circuit technologies in sub$\mathrm{GHz}$ bands. Only references [7], [10], [12] provide some simple line-of-sight demonstrations. However, an adaptive analog weight calculation is essential to support real-world multi-path scenarios, and this was not addressed. Recently, a few hybrid beamforming receivers have been presented at millimeter-wave frequencies in [20]-[24].

In this paper, we propose a hybrid analog-digital beamforming MIMO approach that can adaptively mitigate interference problems in both the analog and digital domain. This requires a multi-beam analog beamformer as in [4] and a digital back-end to perform digital beamforming. Our previous research papers [4], [25], [26] only present the implementation and characterization of the proposed multi-beam analog beamformer without adaptive signal processing. For the system-level EVM measurements demonstrated in [25], [26], the beamforming weights have been manually set assuming a simple line-ofsight scenario and known angle-of-arrivals for the desired signal and interferer. Moreover, the effect of the ADC's quantization noise has not been studied in [25], [26]. In this paper, a control algorithm is proposed for adaptively rejecting the interference while receiving all of the wanted signals from all directions. This improves the signal-to-interference (SIR) ratio, resulting in an interference robust RF/analog front-end. Alternatively, it relaxes the dynamic range requirements for the ADCs, so that power-efficient, low-resolution ADCs suffice for analog-to-digital conversion. This is the main advantage of a hybrid beamforming receiver over a fully digital beamforming receiver. Rejecting the interferer already in the analog domain allows more room to apply a higher gain to suppress the quantization noise of the ADCs. We first validate the concept of an adaptive solution for interference rejection utilizing a MIMO Rx chip and well-defined conductive RF channel measurements. Performing Over-the-Air (OTA) experiments, we demonstrate that it also helps for realistic scenarios. Extensive experimental results show the effectiveness of interference suppression. We do this using two criteria: 1) interference rejection before the ADCs, i.e., the amount of interference rejection in the analog domain; and 2) Error Vector Magnitude (EVM) that takes into account the performance of the whole analog-digital system. EVM is a powerful metric to evaluate link performance, e.g., distinguish noise and distortion effects [26], or to evaluate the coverage range for a $5 \mathrm{G}$ millimeterwave link [27].

The paper is structured as follows. In section II, we summarize the properties of our hybrid beamforming architecture with a brief summary of our analog front-end chip [4], followed by the description of the digital back-end. In section III, we explain the adaptive spatial interference filtering technique.

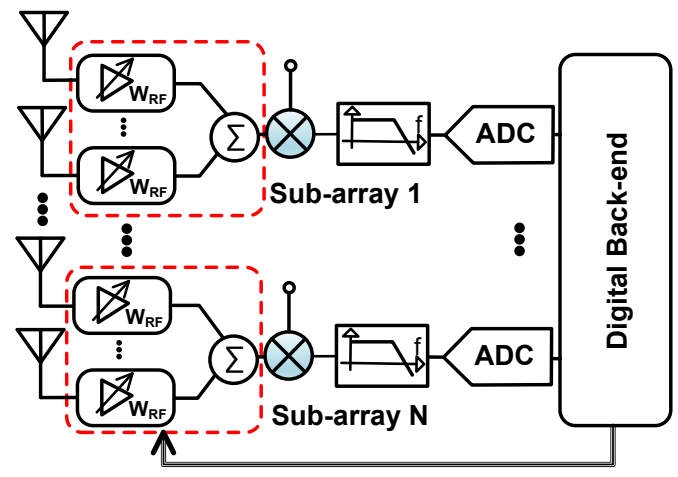

(a)

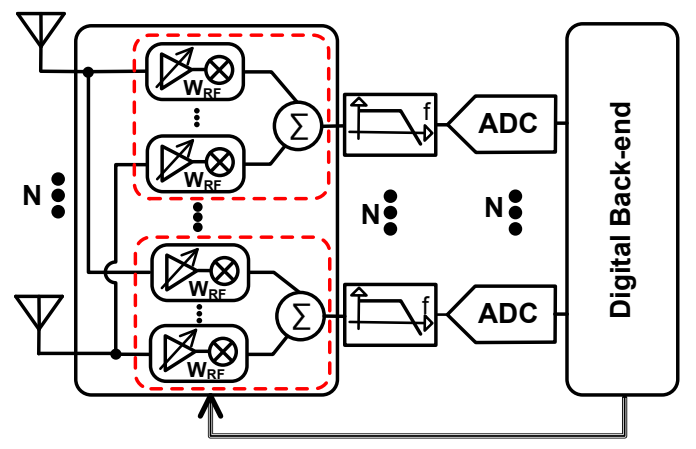

(b)

Fig. 2. (a) Conventional hybrid beamforming at sub-array level to reduce ADC count, i.e., the number of antennas is much higher than N; (b) Proposed hybrid analog-digital beamforming for interference mitigation with the number of antennas and ADCs equal to $N$.

Then, we verify this algorithm using conductive measurement in section IV and expose the MIMO receiver to interference to demonstrate its effectiveness in interference mitigation. We will demonstrate how hybrid beamforming can adaptively address the interference problem and validate this by extensive OTA experiments. Finally, we draw conclusions in section V.

\section{Hybrid BEAMfORMing RECEIVER}

\section{A. Architecture}

Hybrid beamforming MIMO transceiver architectures have been proposed that are mainly aimed at large-scale antenna arrays and recently for massive MIMO applications [28]-[32]. Fig. 2a shows the conventional hybrid beamforming where $\mathrm{RF} / \mathrm{analog}$ beamforming is performed at sub-array level to reduce the number of RF/analog chains, e.g., downconversion mixers and baseband circuitry, and more importantly the number of the ADCs. Complexity reduction in training overhead, i.e., determining channel state information (CSI) between each transmit and receive antenna, is another advantage. These benefits, however, come at the cost of less flexibility in digital signal processing. A recent survey on hybrid beamforming for massive MIMO can be found in [33]. With the hybrid beamforming MIMO receiver, shown in Fig. 2b, we target rejecting strong co-channel interference effectively in a mixed analog digital architecture. The number of antenna elements is equal to the number of ADCs digitizing the same number of analog beamformed outputs, such that each output has contributions from all individual antenna signals. Because 
reconstruction of individual antenna signals is possible in the digital domain, this architecture supports full MIMO functionality but with the added benefit of interference suppression already in the analog domain. To do so, the digital back-end has to adaptively control the analog pre-processing front-end by performing online channel estimation while optimizing the analog beamformer weights.

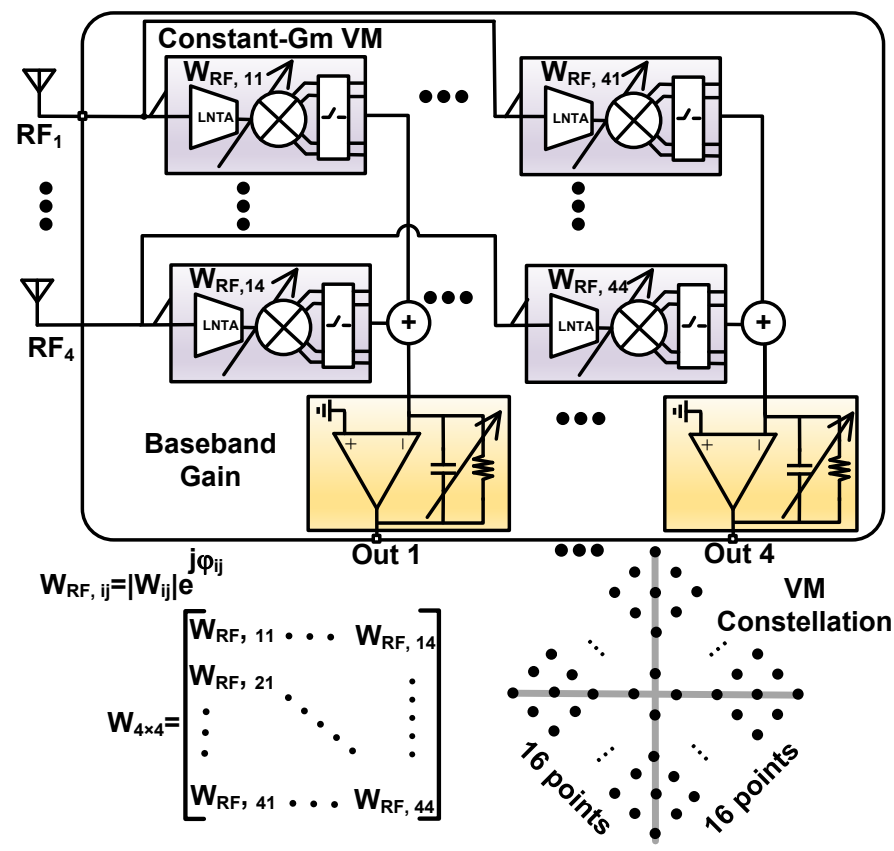

Fig. 3. A 4-element RF/analog front-end consisting of 16 Vector Modulators (VMs) realizing a $4 \times 4$ analog pre-processor [4]. The rotated square constellation includes $16 \times 16$ equidistant points.

\section{B. Analog front-end}

Our receiver front-end described in [4] features an interference robust circuit implementation for hybrid beamforming. The front-end, as shown in Fig. 3, implements a $4 \times 4$ preprocessing matrix where analog complex weights are realized using a "constant-Gm vector modulator" [4], [19]. The rotated constellation square of an individual VM includes $16 \times 16$ equidistant points, allowing for flexible and programmable reconfiguration of the front-end. Because of direct current domain beamforming, this architecture results in a highly linear $\mathrm{RF}$ receiver front-end with wideband interference rejection, as detailed in [4]. Moreover, it achieves a high output referred intercept point (OIP3), a performance metric that is highly relevant for receiver dynamic range.

In [4], we have explained the functionality of our front-end using a simple intuitive example where we program weights to produce a set of orthogonal beams. The idea is to enable reception of signals from all directions, but manipulate their strength so that a strong interferer is suppressed in all of the outputs except for the one that points a beam towards the strong interferer. We have assumed that the interferer's angleof-arrival is known a priori in a simple line-of-sight scenario. In this paper, however, we target smart adaptive approaches that are effective for real-word scenarios where the interferer's angle-of-arrival is unknown.

\section{Adaptive Spatial Interference Nulling TECHNIQUE}

\section{A. System Model}

Consider an uplink MIMO communication system where the receiver is equipped with $N$ antennas and communicates with a single-antenna user (Fig. 4). In addition, co-channel interference severely affects the received signal by transmitting data on the same carrier frequency as the desired user. If the narrowband condition holds for all propagation delays, the equivalent discrete-time data can be modeled as:

$$
\mathbf{x}[k]=\mathbf{h} s[k]+\mathbf{g} i[k]+\mathbf{n}[k]
$$

where the vector $\mathbf{h}=\left[h_{1} h_{2} \ldots h_{N}\right]^{T}$ represents the flat-fading data channel while $\mathbf{g}=\left[g_{1}, g_{1} \ldots g_{N}\right]^{T}$ describes the channel between the interferer and the receiver. The desired user and interference symbols are denoted by $s[k]$ and $i[k]$, respectively. $\mathbf{n}[k]$ is an $N \times 1$ vector and represents additive white Gaussian noise (AWGN) with zero mean and $E\left[\mathbf{n n}^{H}\right]=N_{0} \mathbf{I}$ where $N_{0}$ is the noise variance and $\mathbf{I}$ is an indentity matrix. In general, an $N$-element MIMO receiver can reject $N-1$ interferers.

As previously stated, we evaluated the proposed hybrid beamforming in two different scenarios, conductive and overthe-air (OTA). Therefore, the channels have been modeled in two different ways corresponding to the scenarios. In the conductive scenario, the desired user channel contains a single dominant path, given by

$$
\mathbf{h}=\left[1, e^{j \Phi}, \ldots, e^{j(N-1) \Phi}\right]^{T}
$$

where $\Phi=\frac{2 \pi d}{\lambda} \cos (\phi)$ represents the phase difference between two adjacent antennas signals, $\phi$ is the angle of arrival (AoA), $d$ is the antenna separation distance, and $\lambda$ denotes the carrier wavelength. Similar to the desired user, the interferer channel can be modeled as

$$
\mathbf{g}=\left[1, e^{j \Theta}, \ldots, e^{j(N-1) \Theta}\right]^{T}
$$

where $\Theta=\frac{2 \pi d}{\lambda} \cos (\theta)$ is the inter-antenna phase difference, and $\theta$ represents AoA of the interference signal. As direct and reflected paths affect the channel characterization in the OTA scenario, we consider multi-path channel modeling for both the desired user and the interferer. The data-channel vector is represented as

$$
\mathbf{h}=\sum_{\ell=1}^{L} \alpha_{\ell} \mathbf{a}\left(\phi_{\ell}\right)
$$

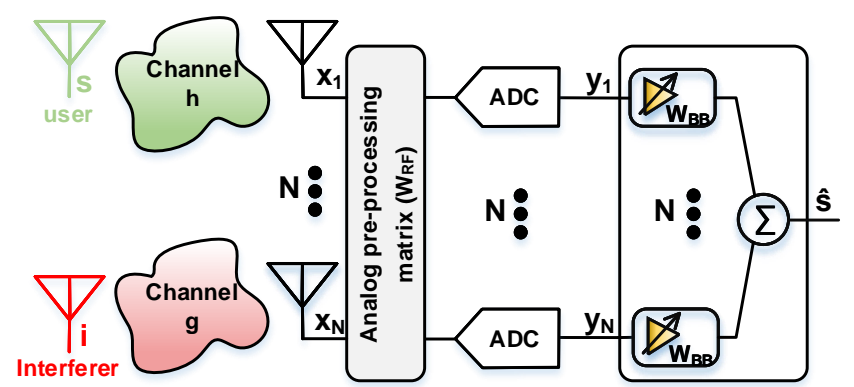

Fig. 4. A single-user uplink MIMO communication system where the receiver is exposed to a strong uplink user in a nearby cell. 
where $L$ is the number of paths, $\alpha_{\ell}$ is the complex gain of the $\ell^{t h}$ path, $\mathbf{a}\left(\phi_{\ell}\right)=\left[1, e^{j\left(\frac{2 \pi d}{\lambda} \cos \left(\phi_{\ell}\right)\right)}, \ldots, e^{j(N-1)\left(\frac{2 \pi d}{\lambda} \cos \left(\phi_{\ell}\right)\right)}\right]^{T}$ denotes the antenna array response between the desired user and the receiver, and $\phi_{\ell}$ shows the angle of arrival of the $\ell^{t h}$ path. The interferer-channel is given by

$$
\mathbf{g}=\sum_{\ell=1}^{L} \beta_{\ell} \mathbf{a}\left(\theta_{\ell}\right)
$$

where $\beta_{\ell}$ denotes the complex gain of the $\ell^{t h}$ path, and the antenna array response between the interferer and the receiver is represented by $\mathbf{a}\left(\theta_{\ell}\right)$.

The hybrid beamforming system described in the previous section consists of an $N \times N$ analog pre-processing matrix $\left(\mathbf{W}_{\mathbf{R F}}\right)$, a group of $N \mathrm{RF}$ chains, and an $N \times 1$ digital beamformer vector. The analog beamformer nulls the interference and maps the antenna signals to $N$ data streams. They are further decoupled by the digital beamformer to decode the desired user data, denoted by $\hat{s}$. Given the analog preprocessing matrix, the discretized and downconverted analog outputs can be written as:

$$
\mathbf{y}[k]=\mathbf{W}_{\mathbf{R F}}^{H} \mathbf{x}[k] .
$$

Each entry of $\mathbf{W}_{\mathbf{R F}}$ is a complex number representing a setting of an analog RF vector modulator. The final post-beamforming signal is obtained by applying the digital beamformer:

$$
\hat{s}[k]=\mathbf{w}_{\mathbf{B B}}^{H} \mathbf{y}[k] .
$$

\section{B. Digital Beamformer Design}

For the given $\mathbf{W}_{\mathbf{R F}}$, the digital beamformer is determined by applying the minimum mean square error (MMSE) estimator:

$$
\begin{gathered}
\mathbf{w}_{\mathbf{B B}}=\underset{\mathbf{w}_{\mathbf{B B}}}{\arg \min } E\left\|s[k]-\mathbf{w}_{\mathbf{B B}}{ }^{H} \mathbf{y}[k]\right\|^{2} \\
\mathbf{w}_{\mathbf{B B}}=\mathbf{R}_{\mathbf{y}}^{-1} \mathbf{r}_{\mathbf{y s}}
\end{gathered}
$$

where $\mathbf{R}_{\mathbf{y}}=E\left\{\mathbf{y}[k] \mathbf{y}^{H}[k]\right\}$ and $\mathbf{r}_{\mathbf{y s}}=E\left\{\mathbf{y}[k] s^{H}[k]\right\}$. Determining $\mathbf{w}_{\mathbf{B B}}$ requires access to training (pilot) signals and all antenna outputs, which is possible thanks to the $N$ data streams produced by the analog front-end. Calculating $\mathbf{W}_{\mathbf{B B}}$ is relatively straightforward when $\mathbf{W}_{\mathbf{R F}}$ is given. Therefore, the main focus is on designing the analog pre-processing part.

\section{Analog Pre-processor Design Based on MMSE Algorithm}

This sub-section describes how to set the analog beamformer weights by utilizing the MMSE beamformer targeting to minimize the mean squared error between the desired user data and the digital beamformer's output. $\mathbf{W}_{\mathbf{R F}}$ is quantized and can be selected from a discrete set of vector modulator settings, a set of complex coefficients with different phases and amplitudes. We also assume high-resolution ADCs to ensure the quantization error is not the dominant limiting factor in the design.

The problem is to find the settings for both the analog and digital beamformer that minimize the error between the training (pilot) signals and the received signal after analog and digital beamforming. Therefore, the problem can be formulated as follow:

$$
\mathbf{W}_{\mathbf{R F}}=\underset{\mathbf{w}_{\mathbf{B B}}, \mathbf{W}_{\mathbf{R F}} \in D^{N}}{\arg \min } \quad E\left\|s[k]-\mathbf{W}_{\mathbf{B B}}{ }^{H} \mathbf{W}_{\mathbf{R F}}{ }^{H} \mathbf{x}[k]\right\| \|^{2}
$$

where $D$ is a dictionary, containing all possible $N \times 1$ vectors that each column of $\mathbf{W}_{\mathbf{R F}}$ can take, and $D^{N}$ represents the set of all possible combinations of settings for $N$ vector modulators. To solve (9), the quantized matching pursuit algorithm [6] has been chosen. It is a greedy technique that recursively selects a vector from the dictionary to find the input vector's best approximation. By taking the steps described in [6], each column of matrix $\mathbf{W}_{\mathbf{R F}}$ can be determined as:

$$
\underline{\mathbf{w}}_{\mathbf{R F}_{i}}=\arg \max _{\underline{\mathbf{w}}_{\mathbf{R F}_{i}} \in \underline{\mathrm{D}}} \frac{\left|\underline{\mathbf{w}}_{\mathbf{R F}}^{H} \underline{\mathbf{r}}_{\mathbf{x s}}\right|}{\left\|\underline{\mathbf{w}}_{\mathbf{R F}_{i}}\right\|}
$$

where, $\underline{\mathbf{w}}_{\mathbf{R F}_{i}}=\mathbf{R}_{\mathbf{x}}{ }^{1 / 2} \mathbf{W}_{\mathbf{R F}}, \underline{\mathbf{r}}_{\mathbf{x s}}=\mathbf{R}_{\mathbf{x}}{ }^{-1 / 2} \mathbf{r}_{\mathbf{x s}}$ and $\underline{\mathrm{D}}=$ $\mathbf{R}_{\mathbf{x}}{ }^{1 / 2} D$. This algorithm requires exhaustive search through the dictionary to obtain each column of $\mathbf{W}_{\mathbf{R F}}$.

\section{Impact of the ADC resolution}

Quantization errors in the ADCs can be modeled as follows:

$$
\mathbf{y}=\mathbf{y}_{q}[k]+\mathbf{e}_{q}[k] .
$$

For uniformly quantized signals $e_{q}$ is within half LSB of the ADC. The total quantization noise power is given by:

$$
P_{q}=\frac{1}{12} \Delta^{2}
$$

where $\Delta$ denotes the LSB size. The effective input-referred quantization noise within the bandwidth of the desired signal can be obtained as follows:

$$
P_{q, \text { input-referred }}[\mathrm{dBm}]=P_{q}[\mathrm{dBm}]+10 \log \left(\frac{2 B W}{f_{S}}\right)-\operatorname{Gain}[\mathrm{dB}]
$$

where $B W, f_{S}$, and Gain are the bandwidth of the desired signal, sampling rate of the ADCs, and the total gain of the front-end, respectively. For a given ADC resolution and sampling rate $\left(f_{S}\right)$, the gain of the front-end has to be high enough to avoid the quantization noise dominating over the thermal noise of the front-end. This is standard practice for digital arrays as well. Thermal noise is usually set to be at approximately one bit above the quantization noise floor of the ADC in fully-digital arrays.

\section{EXPERIMENTAL RESULTS}

\section{A. Conductive measurements}

Measurements were performed to obtain EVM plots versus signal input power to evaluate the effectiveness of spatial filtering. The conductive measurement setup, as shown in Fig. 5 , is used to evaluate the system performance in a controlled fashion. An interference signal emulating a wavefront arriving from spatial angles of $-90^{\circ}$ to $+90^{\circ}$ is produced using external phase shifters. A desired signal having a broadside angle-of-arrival is emulated using power splitters. The differential receiver outputs are sensed using four active probes 


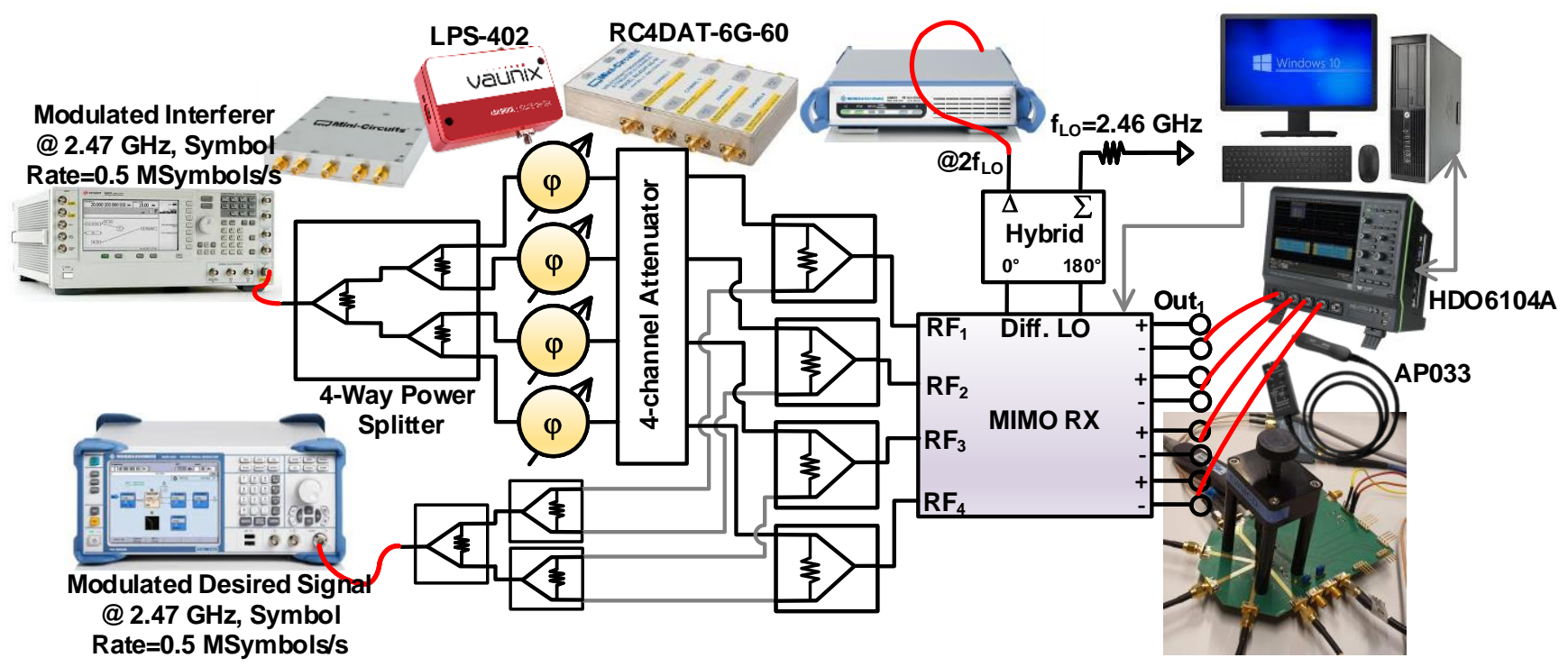

Fig. 5. Conductive measurement set-up of a 4-element antenna emulation system using external phase shifters. One interferer having a variable spatial angle and a desired signal at fixed broadside direction are applied to the MIMO Rx chip.

and applied to a 4-channel High Definition Oscilloscope (HDO6104A) for data acquisition and digital signal processing in MATLAB. To simplify the measurements only the I-outputs are used, and the receiver front-end is set up as a low-IF system operating at $f_{I F}=10 \mathrm{MHz}$. A single carrier 0.5MSymbols/s QPSK desired signal at $f_{R F}=2.47 \mathrm{GHz}$ and a root cosine pulse shaping filter with $\beta=0.35$ is generated while a Pseudo Random Binary Sequence (PRBS) data was used. We have chosen a low symbol rate of 0.5 -MSymbols/s to simplify over-the-air measurements as it does not require channel equalization techniques. The efficacy of interference suppression for wideband interference can be affected by the delay-phase approximation [18], which holds for narrowband systems. However, in [25], we have experimentally demonstrated that our MIMO Rx front end can effectively reject wideband interference with a symbol rate as high as 50 MSymbols/s. For QPSK digital demodulation, the following steps were taken: After frequency downconversion and low pass filtering, symbol timing synchronization is performed using the Gardner's timing error detector [34]. Then, a Costas loop [34] is used to perform carrier phase recovery. Finally, frame synchronization to find the first symbol is done by crosscorrelation.

In our first experiment, the signal power is swept and EVM is measured. The resulting EVM plot is shown in Fig. 6. As explained in [26], there are 3 regions that we refer to as 1) noise-limited; 2) best EVM; and 3) distortion-limited regions. In the noise-limited region, the SNR in $\mathrm{dB}$ is related to the $\mathrm{EVM}$ as $S N R=-20 \log (E V M(\%) / 100)$ assuming additive white Gaussian noise such as the receiver's thermal noise. This region can be affected by the quantization noise as well. EVM in the distortion-limited region, i.e., high signal power levels, depends on the in-band linearity of the receiver (OIP3). OIP3 is almost independent of gain; hence, the front-end gain affects the input power level at which the distortion starts to degrade the EVM.

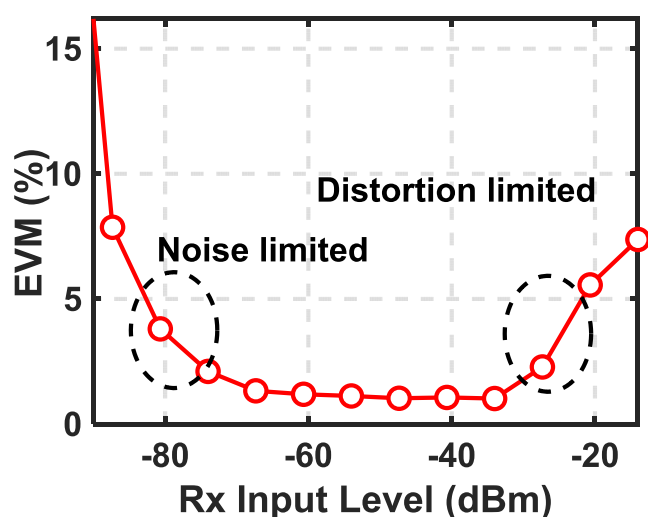

Fig. 6. Measured EVM versus input power for a 0.5-MSymbols/s QPSK signal and Gain $=32 \mathrm{~dB}$.

To verify the effectiveness of the MMSE algorithm, EVM measurements were done in the presence of a co-channel interferer having 0.5-MSymbols/s QPSK modulation at $\mathrm{P}_{\mathrm{int}}=-$ $70 \mathrm{dBm}$ arriving from $+30^{\circ}$. In order to find the weights of the analog preprocessing matrix using MMSE, the channel estimation is done applying a pilot signal with $\mathrm{P}_{\text {sig }}=-55 \mathrm{dBm}$. After finding the settings, EVM versus input power level is measured. For this experiment, the EVM performance at the analog output ( $y_{1}$ in Fig. 4) and the hybrid beamforming output ( $\hat{s}$ in Fig. 4 ) are shown in Fig. 7a. To show the effect of spatial filtering on EVM, results without spatial filtering are also shown. The "best EVM" region is improved by 33 $\mathrm{dB}$. In the "best EVM" region, the desired signal is stronger than the noise and interference power, therefore, the EVM is not limited by SNR or SIR ratio. As a result, both the hybrid beamforming output and the analog beamformed output show the same EVM performance. These experiments were also repeated for an interferer arriving from $+45^{\circ}$, resulting in an improvement of the "best EVM" by $27 \mathrm{~dB}$ as shown in 
Fig. 7b. Output spectrum and constellations for the experiment of Fig. 7a are shown in Fig. 8a and Fig. 8b, respectively.

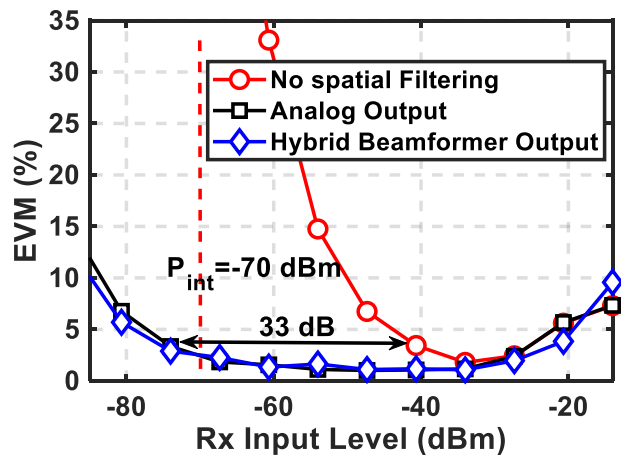

(a)

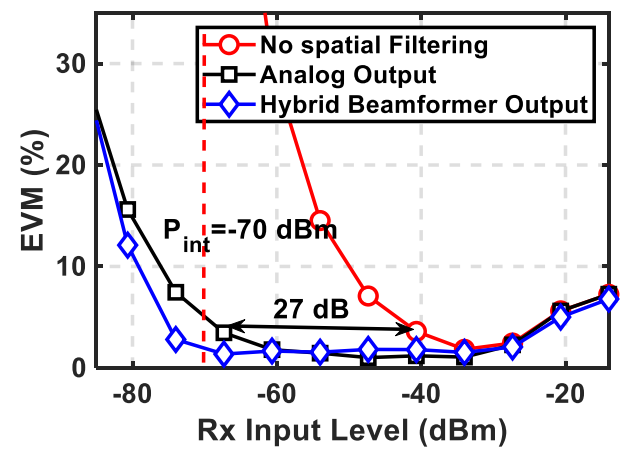

(b)

Fig. 7. Measured EVM in the presence of an interferer after applying spatial filtering by MMSE (a) Interferer is at $+30^{\circ}$; (b) Interferer is at $+45^{\circ}$
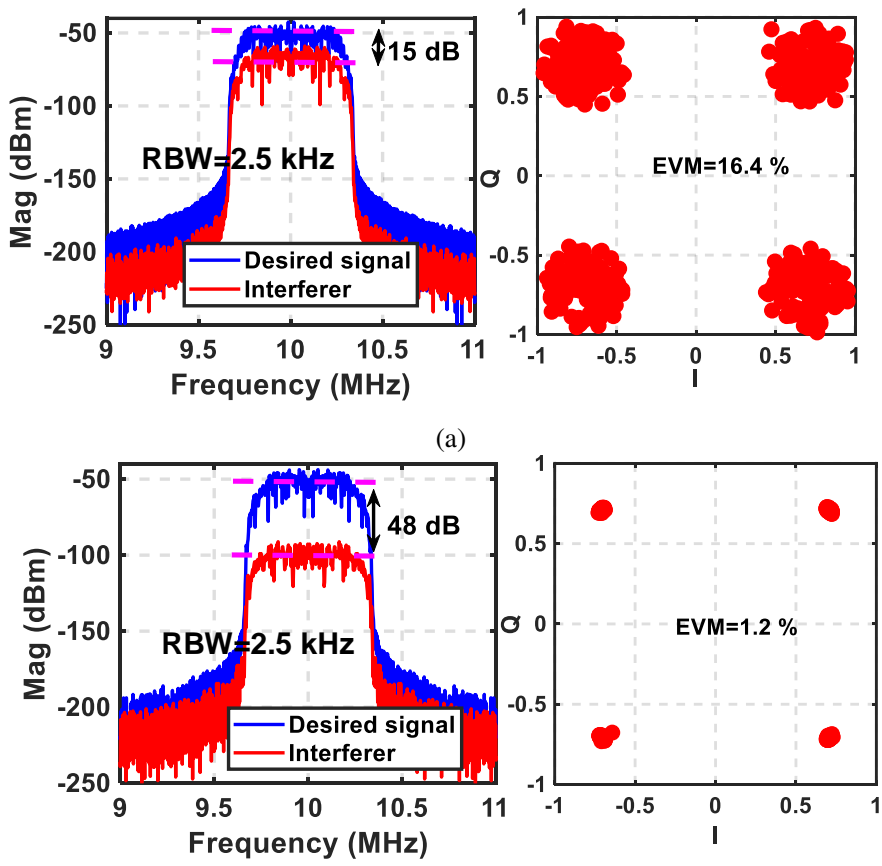

(b)

Fig. 8. Output spectrum and constellation when a 0.5-MSymbols/s QPSK desired signal and a 15-dB weaker interferer are both arriving from broadside (no spatial filtering); (b) Output spectrum and constellation when the desired signal is arriving from broadside and the interferer from a null angle at $30^{\circ}$ (spatial filtering).

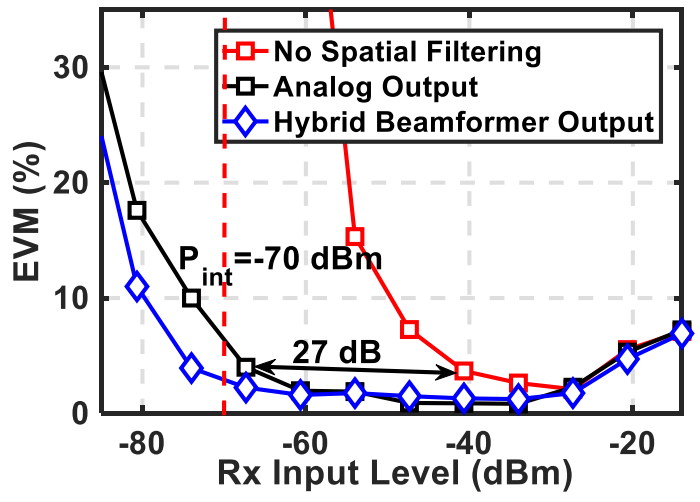

Fig. 9. Measured EVM versus input power in the presence of an interferer at $45^{\circ}$ and $\operatorname{SIR}_{\text {in }}=4 \mathrm{~dB}$ in the training phase.

As we mentioned earlier, the carrier phase synchronization and symbol time recovery are performed using a Costas loop and Gardner's timing error detector where the input SIR is set to $15 \mathrm{~dB}$ to determine the analog beamformer settings. However, in the presence of a stronger interference signal with the same bandwidth as the desired user, none of these methods perform correctly. Using a data-aided method is a possible solution as the pilot tone signals are known in the training phase. Therefore, we employed the zero-crossing timing error detector (ZCTED) [34] for symbol time recovery and the dataaided phase detector [34] for carrier phase synchronization. The applied modification reduced the required input SIR to $4 \mathrm{~dB}$ without degrading the EVM performance. The resulting EVM plot versus input power level is depicted in Fig. 9 where an interference rejection of $27 \mathrm{~dB}$ is achieved.

The data-aided methods still fail to reliably perform the symbol time recovery and carrier phase synchronization in the case of negative SIR, i.e., the interferer is stronger than the desired user. This issue has been investigated in the literature [35]-[37]. Ref. [37] introduces a two-stage MMSE estimator where, as the first stage, a pre-filter reduces the interference to a level that allows for symbol time recovery and carrier phase synchronization. In this paper, we will not further elaborate on this.

To evaluate the effect of quantization noise on the EVM, the captured oscilloscope signals were quantized virtually in MATLAB. We have chosen an ADC input range of $-1 \mathrm{~V}$ to $+1 \mathrm{~V}$. The EVM plots versus receiver input level are shown for various numbers of ADC bits (6 to 12 bits) in Fig. 10a. For the 12-bit resolution, the thermal noise of the oscilloscope and the Rx front-end are dominant, so the quantization noise plays a small role in degrading the EVM. For ADC resolutions ranging from 6 to 10 bits, the quantization noise becomes the dominant source of EVM degradation when lowering the input power level. Note that when the received signal amplitude is less than one LSB, EVM becomes almost flat. It is also evident from the EVM plots that the power level at which the EVM degradation occurs, changes approximately $6 \mathrm{~dB}$ with every ADC bit as expected. The quantization noise can be masked by a high front-end gain. However, the input power upper limit for the "best EVM" region reduces as well, so the overall $\mathrm{Rx}$ 
dynamic range does not change to the first order. This is shown in Fig. $10 \mathrm{~b}$ in case the number of ADC bits equals $\mathrm{n}=8$, EVM plots were measured for gain values ranging from 29 to 41 dB.

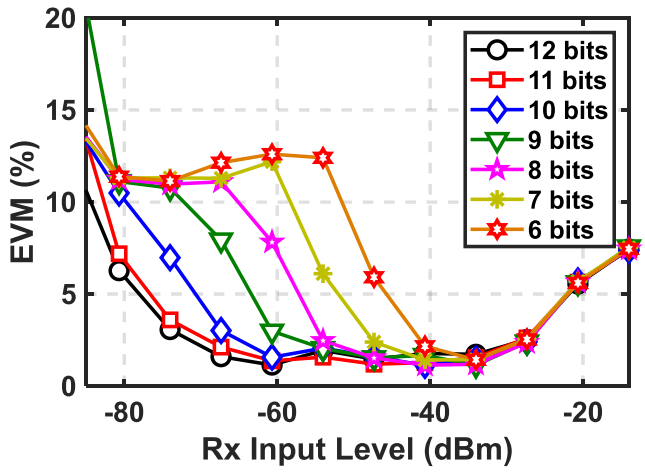

(a)

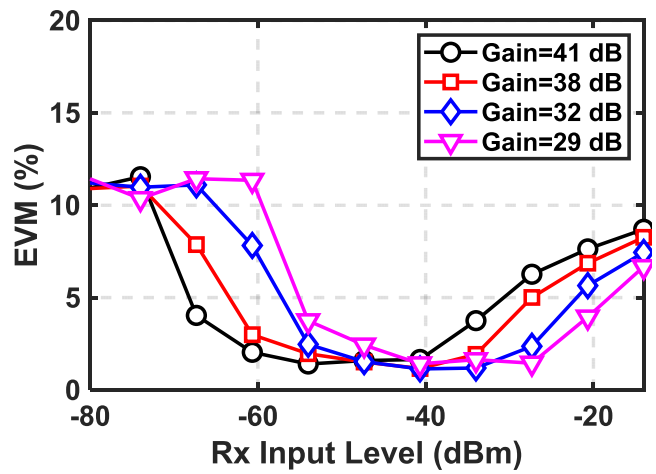

(b)

Fig. 10. EVM versus Rx input level for various number of ADC bits; the total gain of Rx front-end Gain=32 dB; (b) EVM versus Rx input level for various receiver gains and number of ADC bits $\mathrm{n}=8\left(f_{s}=50 \mathrm{MS} / \mathrm{s}\right.$, QPSK modulation)

For interference-free scenarios, a high enough gain can mask the quantization noise. However, in the presence of an in-band adjacent channel interferer, the amount of gain should be limited to avoid distortion at the front-end as well as ADC clipping. The main advantage of a hybrid beamforming receiver over a fully digital beamforming receiver is that rejecting the interferer already in the analog domain allows more room to apply a higher gain to suppress the quantization noise of the ADCs. To show this advantage, a strong adjacent channel interferer with 0.5-Msymbols/s using QPSK modulation and having a power level of $-20 \mathrm{dBm}$ is applied at $f_{R F}=2.471 \mathrm{GHz}$. A 0.5-Msymbols/s QPSK desired signal at $f_{R F}=2.47 \mathrm{GHz}$ is used as in the previous experiments. As shown in Fig. 11, the EVM plots are measured for a single element Rx $($ Gain $=20.5 \mathrm{~dB})$, the fully digital beamforming (Gain=20.5 dB), and hybrid beamforming with a notch set to the interferer's angle-of-arrival (AoA), e.g., $+30^{\circ}$ (AoA is known in this particular experiment), allowing for a gain as high as $41 \mathrm{~dB}$ without experiencing distortions at the RF front-end in the presence of the strong interferer. In order to make sure that the quantization noise is the dominant source of the EVM degradation, the captured oscilloscope signals are quantized assuming a 6-bit ADC. In the quantization noise limited region of Fig. 11, the EVM difference in $\mathrm{dB}$ for a single element $\mathrm{Rx}$ and hybrid beamforming is about 19 $\mathrm{dB}$ (calculated from Fig. 11 at the input power level of -54 $\mathrm{dBm}$, i.e., $20 \log (32.2 / 3.5)=19 \mathrm{~dB})$, which approximately fits to the gain difference of $20.5 \mathrm{~dB}$ between them. For the fully digital beamforming, there is a 6-dB EVM improvement (calculated from Fig. 11 at the input power level of -54 $\mathrm{dBm}$, i.e., $20 \log (32.2 / 16.3) \approx 6 \mathrm{~dB})$ due to the summing of uncorrelated quantization noise of the single Rx elements.

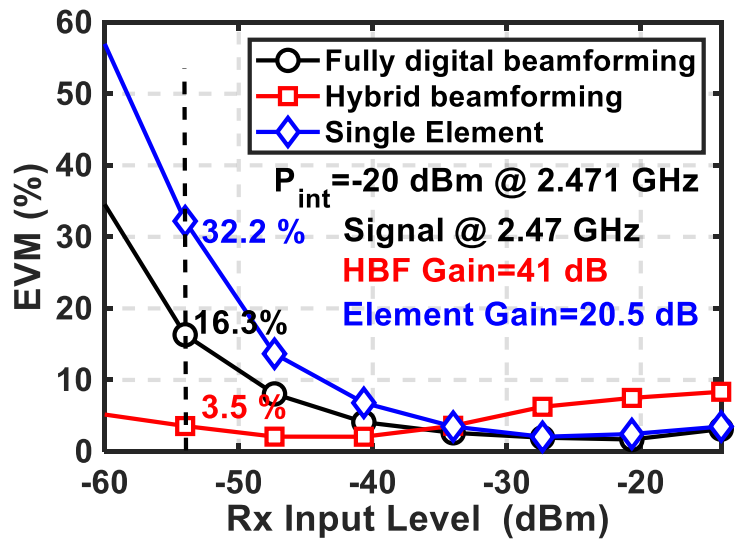

Fig. 11. Measured EVM versus input power level for a 0.5-MSymbols/s QPSK desired signal at $f_{R F}=2.47 \mathrm{GHz}$ in the presence of a 0.5 MSymbols/s QPSK adjacent channel interferer at $f_{R F}=2.471 \mathrm{GHz}$ with input power level of $-20 \mathrm{dBm}$. For hybrid beamforming the notch is set at the interferer's angle-of-arrival, e.g., $+30^{\circ}$.

\section{B. Over-the-air (OTA) measurements}

OTA measurements were done with the set-up shown in Fig. 12 that uses a 4-element dipole antenna array with halfwavelength spacing in the $2.4 \mathrm{GHz}$ ISM-band as the Rx array. For $\mathrm{Tx}$, one antenna transmits the desired signal and one antenna the interferer. Because we are interested in the effectiveness of spatial filtering for a real world scenario, we have performed OTA experiments in the environment as it is. A 0.5MSymbols/s QPSK desired signal and interferer are used. To find the setting of the analog VMs, first, channel estimation is done with an approximately $15-\mathrm{dB}$ weaker interferer than the desired signal. OTA experiments are performed for constantGm VM constellations with 16 and 64 points as shown in Fig. 13. For these experiments, the desired signal transmit antenna is placed at broadside $\left(0^{\circ}\right)$ and the location of the interferer transmit antenna is varied. The rejection results are summarized for a few locations of the interferer in Fig. 14. The EVM values are limited by the residue of the interferer power at the output and therefore, closely related to the rejection amount, i.e., $\mathrm{EVM}[\mathrm{dB}]=-\mathrm{SIR}_{\text {out }}=-\left(\right.$ Rejection $\left.+\mathrm{SIR}_{\text {in }}\right)$. A moderately strong desired signal with the input power level of $-31 \mathrm{dBm}$ and an interferer with the power level of $-37 \mathrm{dBm}$ $\left(\mathrm{SIR}_{\mathrm{in}}=6 \mathrm{~dB}\right)$ are chosen for reliable results in the presence of external interfering signals (crowded ISM band). Because of the multipath propagation, the antenna signals and therefore, the rejection amount varies with relatively small changes in the antenna placements. On average, $22.5 \mathrm{~dB}$ and $24.5 \mathrm{~dB}$ interference rejection are achieved using VMs with 16 and 64 
points, respectively, for 16 different experiments, as shown in Fig. 14.

To verify the measurement results, we evaluate the proposed hybrid beamforming performance by running 200 Monte Carlo simulations. The simulation parameters are set as follows: the antenna array size and the number of RF chains $N=4$, the received SIR and SNR $6 \mathrm{~dB}$ and $60 \mathrm{~dB}$, respectively, Rician channel modeling for both user and interferer with $\mathrm{K}$, the ratio between the power in the direct and in the scattered paths, set to $4 \mathrm{~dB}$, and all path gains follow the independent and identically distributed (i.i.d) Rician. To represent the antennas' mutual coupling effect, we use the coupling matrix obtained by the impedance matrix or $\mathrm{Z}$ approach [38]. The interference rejection is defined as $\operatorname{SIR}_{\text {out }} / \mathrm{SIR}_{\text {in }}$, and the results are obtained by averaging over 200 different channel realizations while transmitting a set of 511 QPSK symbols. Simulation results show $20.5 \mathrm{~dB}$ and $22.8 \mathrm{~dB}$ interference rejection by employing VMs with 16 and 64 points, which are close to the achieved measurement results.

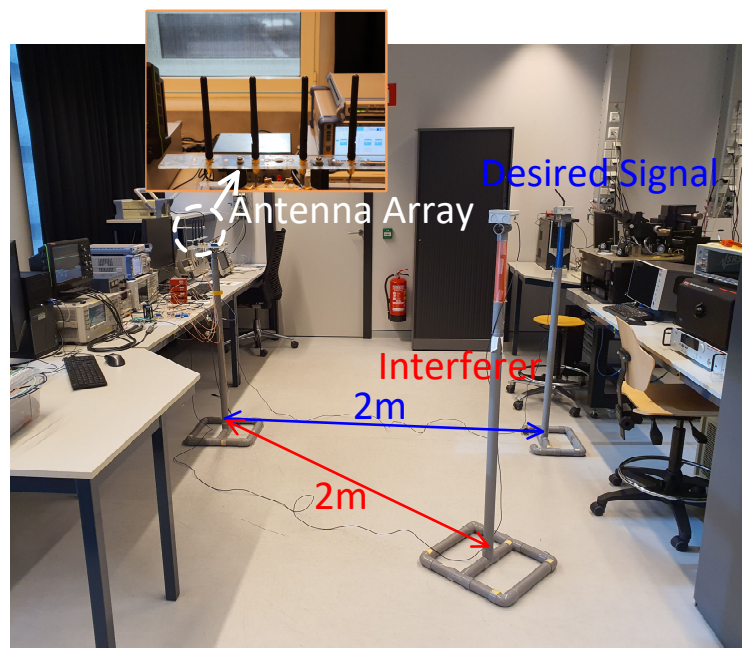

Fig. 12. OTA measurement set up using a 4-element dipole antenna array with half-wavelength spacing in the $2.4 \mathrm{GHz}$ ISM-band.

In this work, our goal is to show the effectiveness of hybrid beamforming in interference mitigation with a prototype MIMO receiver chip in an indoor environment where the multipath propagation effects and the coupling between array elements heavily impact the results. Hence, it is far from straightforward to benchmark these real world results with the literature. Nevertheless, we have summarized the performance of our prototype MIMO Rx chip in Table I and benchmarked it with the recently published MIMO receivers in the low$\mathrm{GHz}$ frequency range. In [12], an OTA experiment has been reported, where a $1 \mathrm{MSymbols/s}$ QPSK desired signal at the input power level of $-68 \mathrm{dBm}$ and two $\mathrm{CW}$ interferers at 600 $\mathrm{kHz}$ frequency offset from the center of the desired signal with the power level of $-57 \mathrm{dBm}$ for each of the interferers were used. In [12], the angle-of-arrivals (AoA) are known for the interferers and the beamforming weights are manually tuned, which results in an EVM of $20.5 \%$ after spatial filtering. Although OTA rejection has not been reported, we estimate a rejection amount of $24.8 \mathrm{~dB}$ for the OTA experiment in

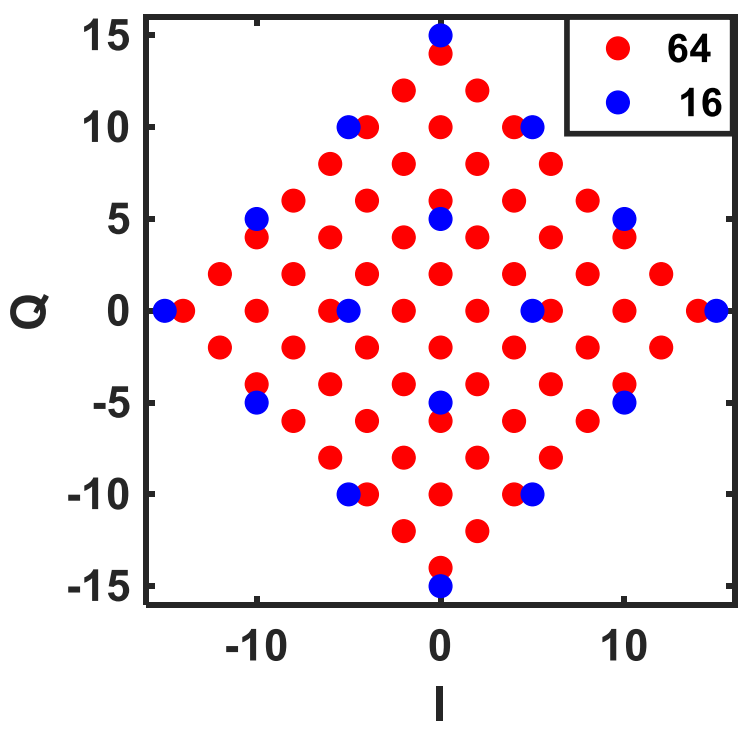

Fig. 13. Constant-Gm VM constellations with 16 and 64 points used for OTA experiments.

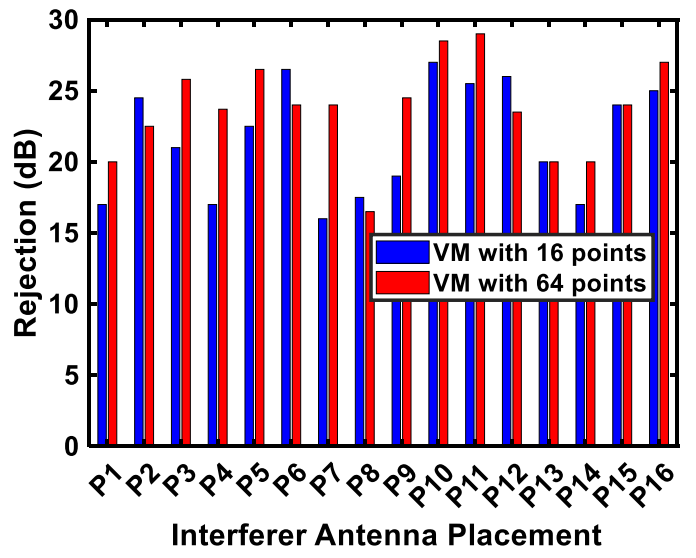

Fig. 14. Measured interference rejection for 16 OTA experiments with different interferer antenna placements and using VMs with 16 and 64 points.

[12] based on the reported EVM value and the input power levels. For the wireless imaging demonstration in [10], which includes one $\mathrm{CW}$ desired signal and one in-band $\mathrm{CW}$ blocker, a rejection of $29.7 \mathrm{~dB}$ was reported.

To relate the OTA results to the conductive measurement results, note that the achievable interference rejection in a hybrid beamforming receiver represents the reduction in the required dynamic range for the ADCs when receiving a weak desired signal in the presence of a strong adjacent-channel interferer as in Fig. 11, because by applying interference rejection in the analog domain before the ADCs, more gain (equal to the rejection) can be allowed without the EVM performance being limited by distortion. The dynamic range reduction achieved by the hybrid beamforming is equal to the rejection amount when compared with a traditional single antenna receiver and 6-dB less than the rejection amount when compared with the 4-element fully digital MIMO Rx. 
TABLE I

PERFORMANCE SUMMARY AND COMPARISON WITH STATE-OF-THE-ART.

\begin{tabular}{|c|c|c|c|c|}
\hline Architecture & $\begin{array}{c}\text { RFIC'16 } \\
{[8]}\end{array}$ & $\begin{array}{c}\text { JSSC'16 } \\
{[10]}\end{array}$ & $\begin{array}{c}\text { JSSC'17 } \\
\text { [12] }\end{array}$ & This work \\
\hline CMOS Technology & $65 \mathrm{~nm}$ & $65 \mathrm{~nm}$ & $65 \mathrm{~nm}$ & $22 \mathrm{~nm}$ FD-SOI \\
\hline Active area $\left(\mathrm{mm}^{2}\right)$ & 3.8 & 1.69 & 1.44 & 0.52 \\
\hline Supply (V) & $1.3-1.5$ & 1.2 & 1.2 & 0.8 \\
\hline \# of inputs/outputs & $4 / 4$ & $4 / 4$ & $4 / 4$ & $4 / 4$ \\
\hline RF Frequency (GHz) & 10 & $0.1-1.7$ & $0.1-3.1$ & $0.7-5.7$ \\
\hline Total Gain (dB) & 41 & 43 & 44.5 & 41 \\
\hline Single Element $\mathrm{NF}_{\mathrm{DSB}, \mathrm{eq}}(\mathrm{dB})$ & 9.5 & $2.2-4.6$ & $3.4-5.8$ & $\begin{array}{c}6.8-9.2^{1} \\
5.5-7^{2} \\
\end{array}$ \\
\hline Conductive Rejection (dB) & $32^{3}$ & 32 & $51-56$ & $\begin{array}{l}33 @ 30^{\circ} \\
27 @ 45^{\circ}\end{array}$ \\
\hline OTA Rejection (dB) & - & 29.7 & $24.8^{4}$ & $\begin{array}{l}22.5^{5} \\
24.5^{6}\end{array}$ \\
\hline Adaptive Beamforming Method & - & - & - & MMSE \\
\hline Blocker Modulation Scheme & - & $\mathrm{CW}$ & $\mathrm{CW}$ & $0.5 \mathrm{MS} / \mathrm{s}$ QPSK \\
\hline Signal Modulation Scheme & - & $\mathrm{CW}$ & $1 \mathrm{MS} / \mathrm{s}$ QPSK & $0.5 \mathrm{MS} / \mathrm{s}$ QPSK \\
\hline OIP3 (dBm) & - & 0 & 11 & 22 \\
\hline $\begin{array}{l}\text { Power Consumption } \\
\text { /Antenna Element }(\mathrm{mW})\end{array}$ & 145 & $37 @ 0.5 \mathrm{GHz}$ & $29-36.75$ & $19.25-34.75$ \\
\hline
\end{tabular}

${ }^{1}$ On the VM corner and broadside $(\mathrm{u}=0)^{2}$ On the VM corner and endfire $(\mathrm{u}=1)^{3}$ From the comparison table in [12] ${ }^{4}$ Estimated from the EVM and input power levels ${ }^{5}$ Average of 16 experiments with a 16-points $\mathrm{VM}^{6}$ Average of 16 experiments with a 64-points VM.

\section{CONCLUSiON}

In this paper, we have experimentally shown the effectiveness of hybrid analog-digital beamforming in interference mitigation using a 4-element MIMO Rx chip featuring analog beamforming before the ADCs. The hybrid approach has an advantage over a fully digital one because the rejection of a strong interferer already in the analog domain allows more room to apply a higher front-end gain to mask the quantization noise of the ADCs while preventing distortion caused by nonlinear RF/analog front-ends. Conductive measurements were performed to reliably verify the performance of adaptive MMSE in interference rejection and demonstrate the reduction of the required dynamic range for the ADCs in the presence of a strong adjacent channel interferer. In order to verify the results in a realistic setup, over-the-air measurements were done to account for multipath propagation and coupling between the antenna elements. Over-the-air interference rejection values of $22.5 \mathrm{~dB}$ and $24.5 \mathrm{~dB}$ have been measured on average for the vector modulators with 16 and 64 points, respectively.

\section{ACKNOWLEDGMENT}

The authors would like to thank Gerard Wienk for CAD assistance, Arnoud Rop for measurement set-up, and Bram Nauta for discussions. The authors also thank GlobalFoundries for supporting chip fabrication.

\section{REFERENCES}

[1] N. Peccarelli, B. James, R. Irazoqui, J. Metcalf, C. Fulton, and M. Yeary, "Survey: Characterization and Mitigation of Spatial/Spectral Interferers and Transceiver Nonlinearities for 5G MIMO Systems," IEEE Transactions on Microwave Theory and Techniques, vol. 67, no. 7, pp. 28292846, July 2019.
[2] L. Zhang and H. Krishnaswamy, "A scalable $0.1-$ to- $1.7 \mathrm{GHz}$ spatiospectral-filtering 4-element MIMO receiver array with spatial notch suppression enabling digital beamforming," in IEEE Int. Solid-State Circuits Conf. (ISSCC) Dig. Tech. Papers, Jan. 2016, pp. 166-167.

[3] — "A 0.1-to-3.1GHz 4-element MIMO receiver array supporting analog/RF arbitrary spatial filtering," in IEEE Int. Solid-State Circuits Conf. (ISSCC) Dig. Tech. Papers, Feb. 2017, pp. 410-411.

[4] S. Golabighezelahmad, E. Klumperink, and B. Nauta, "A 1-4 GHz 4x4 MIMO Receiver with 4 Reconfigurable Orthogonal Beams for Analog Interference Rejection," in IEEE Radio Frequency Integrated Circuits Symp. (RFIC), June 2019, pp. 339-342.

[5] J. H. C. van den Heuvel, J. P. M. G. Linnartz, P. G. M. Baltus, and D. Cabric, "Full MIMO Spatial Filtering Approach for Dynamic Range Reduction in Wideband Cognitive Radios," IEEE Trans. Circuits Syst. I, vol. 59, no. 11, pp. 2761-2773, Nov. 2012.

[6] V. Venkateswaran and A. van der Veen, "Analog Beamforming in MIMO Communications With Phase Shift Networks and Online Channel Estimation," IEEE Transactions on Signal Processing, vol. 58, no. 8, pp. 4131-4143, Aug 2010.

[7] R. W. Irazoqui and C. J. Fulton, "Spatial Interference Nulling Before RF Frontend for Fully Digital Phased Arrays," IEEE Access, vol. 7, pp. $151261-151272,2019$.

[8] S. Jain, Y. Wang, and A. Natarajan, "A $10 \mathrm{GHz}$ CMOS RX frontend with spatial cancellation of co-channel interferers for MIMO/digital beamforming arrays," in IEEE Radio Frequency Integrated Circuits Symp., May 2016, pp. 99-102.

[9] H. Krishnaswamy and L. Zhang, "Analog and RF Interference Mitigation for Integrated MIMO Receiver Arrays," Proc. IEEE, vol. 104, no. 3, pp. 561-575, March 2016.

[10] L. Zhang, A. Natarajan, and H. Krishnaswamy, "Scalable Spatial Notch Suppression in Spatio-Spectral-Filtering MIMO Receiver Arrays for Digital Beamforming," IEEE J. Solid-State Circuits, vol. 51, no. 12, pp. 3152-3166, Dec. 2016.

[11] C. Kim, S. Joshi, C. M. Thomas, S. Ha, L. E. Larson, and G. Cauwenberghs, "A $1.3 \mathrm{mw} 48 \mathrm{mhz} 4$ channel mimo baseband receiver with 65 $\mathrm{db}$ harmonic rejection and $48.5 \mathrm{db}$ spatial signal separation," IEEE $J$. Solid-State Circuits, vol. 51, no. 4, pp. 832-844, April 2016.

[12] L. Zhang and H. Krishnaswamy, "Arbitrary Analog/RF Spatial Filtering for Digital MIMO Receiver Arrays," IEEE J. Solid-State Circuits, vol. 52, no. 12, pp. 3392-3404, Dec. 2017.

[13] J. Paramesh, R. Bishop, K. Soumyanath, and D. Allstot, "A 1.4V $5 \mathrm{GHz}$ four-antenna Cartesian-combining receiver in $90 \mathrm{~nm}$ CMOS for 
beamforming and spatial diversity applications," in IEEE Int. Solid-State Circuits Conf. (ISSCC) Dig. Tech. Papers, vol.1, Feb. 2005, pp. 210-594.

[14] R. Tseng, H. Li, D. H. Kwon, Y. Chiu, and A. S. Y. Poon, "A Four-Channel Beamforming Down-Converter in 90-nm CMOS Utilizing Phase-Oversampling," IEEE J. Solid-State Circuits, vol. 45, no. 11, pp. 2262-2272, Nov. 2010.

[15] M. C. M. Soer, E. A. M. Klumperink, B. Nauta, and F. E. van Vliet, "A 1.0 -to- $4.0 \mathrm{GHz} 65 \mathrm{~nm}$ CMOS four-element beamforming receiver using a switched-capacitor vector modulator with approximate sine weighting via charge redistribution," in IEEE Int. Solid-State Circuits Conf. (ISSCC) Dig. Tech. Papers, Feb. 2011, pp. 64-66.

[16] A. Ghaffari, E. A. M. Klumperink, F. E. van Vliet, and B. Nauta, "Simultaneous spatial and frequency-domain filtering at the antenna inputs achieving up to $+10 \mathrm{dBm}$ out-of-band/beam P1dB," in IEEE Int. Solid-State Circuits Conf. (ISSCC) Dig. Tech. Papers, Feb. 2013, pp. 84-85.

[17] M. C. M. Soer, E. A. M. Klumperink, B. Nauta, and F. E. van Vliet, "1.0to- $2.5 \mathrm{GHz}$ beamforming receiver with constant-Gm vector modulator consuming $<9 \mathrm{~mW}$ per antenna element in $65 \mathrm{~nm}$ CMOS," in ISSCC, Feb. 2014, pp. 66-67.

[18] S. K. Garakoui, E. A. M. Klumperink, B. Nauta, and F. E. van Vliet, "Compact Cascadable g m -C All-Pass True Time Delay Cell With Reduced Delay Variation Over Frequency," IEEE J. Solid-State Circuits, vol. 50, no. 3, pp. 693-703, March 2015.

[19] M. C. M. Soer, E. A. M. Klumperink, D. van den Broek, B. Nauta, and F. E. van Vliet, "Beamformer With Constant-Gm Vector Modulators and Its Spatial Intermodulation Distortion," IEEE J. Solid-State Circuits, vol. 52, no. 3, pp. 735-746, March 2017.

[20] S. Mondal, R. Singh, A. I. Hussein, and J. Paramesh, "A $25-30 \mathrm{GHz}$ Fully-Connected Hybrid Beamforming Receiver for MIMO Communication," IEEE J. Solid-State Circuits, vol. 53, no. 5, pp. 1275-1287, 2018.

[21] S. Mondal and J. Paramesh, "A Reconfigurable 28-/37-GHz MMSEAdaptive Hybrid-Beamforming Receiver for Carrier Aggregation and Multi-Standard MIMO Communication," IEEE J. Solid-State Circuits, vol. 54, no. 5, pp. 1391-1406, 2019.

[22] _ _Power-Efficient Design Techniques for mm-Wave Hybrid/Digital FDD/Full-Duplex MIMO Transceivers," IEEE J. Solid-State Circuits, vol. 55, no. 8, pp. 2011-2026, 2020.

[23] M. Huang and H. Wang, "A Mm-Wave Wideband MIMO RX With Instinctual Array-Based Blocker/Signal Management for Ultralow-Latency Communication," IEEE J. Solid-State Circuits, vol. 54, no. 12, pp. $3553-$ 3564, 2019.

[24] M. Huang, T. Chi, F. Wang, T. Li, and H. Wang, "A Full-FoV Autonomous Hybrid Beamformer Array With Unknown Blockers Rejection and Signals Tracking for Low-Latency 5G mm-Wave Links," IEEE Transactions on Microwave Theory and Techniques, vol. 67, no. 7, pp. 2964-2974, 2019.

[25] S. Golabighezelahmad, E. A. M. Klumperink, and B. Nauta, "A 0.7-5.7 GHz Reconfigurable MIMO Receiver Architecture for Analog Spatial Notch Filtering Using Orthogonal Beamforming," IEEE J. Solid-State Circuits, doi: 10.1109/JSSC.2020.3028811.

[26] S. Golabighezelahmad, E. Klumperink, and B. Nauta, "EVM-based Performance Evaluation of Co-channel Interference Mitigation using Spatial Filtering for Digital MIMO Receivers," in 2020 IEEE 92nd Vehicular Technology Conference (VTC2020-Fall), 2020, pp. 1-5.

[27] M. E. Leinonen, M. Jokinen, N. Tervo, O. Kursu, and A. Pärssinen, "System EVM Characterization and Coverage Area Estimation of 5G Directive mmW Links," IEEE Transactions on Microwave Theory and Techniques, vol. 67, no. 12, pp. 5282-5295, Dec. 2019.

[28] Xinying Zhang, A. F. Molisch, and Sun-Yuan Kung, "Variable-phaseshift-based RF-baseband codesign for MIMO antenna selection," IEEE Transactions on Signal Processing, vol. 53, no. 11, pp. 4091-4103, Nov. 2005.

[29] J. Ahmadi-Shokouh, S. H. Jamali, and S. Safavi-Naeini, "Optimal receive soft antenna selection for MIMO interference channels," IEEE Transactions on Wireless Communications, vol. 8, no. 12, pp. 58935903, Dec. 2009.

[30] O. E. Ayach, S. Rajagopal, S. Abu-Surra, Z. Pi, and R. W. Heath, "Spatially Sparse Precoding in Millimeter Wave MIMO Systems," IEEE Transactions on Wireless Communications, vol. 13, no. 3, pp. 14991513, March 2014.

[31] A. Alkhateeb, O. El Ayach, G. Leus, and R. W. Heath, "Channel Estimation and Hybrid Precoding for Millimeter Wave Cellular Systems," IEEE Journal of Selected Topics in Signal Processing, vol. 8, no. 5, pp. 831-846, Oct 2014.
[32] L. Liang, W. Xu, and X. Dong, "Low-Complexity Hybrid Precoding in Massive Multiuser MIMO Systems," IEEE Wireless Communications Letters, vol. 3, no. 6, pp. 653-656, Dec 2014.

[33] A. F. Molisch, V. V. Ratnam, S. Han, Z. Li, S. L. H. Nguyen, L. Li, and K. Haneda, "Hybrid Beamforming for Massive MIMO: A Survey," IEEE Communications Magazine, vol. 55, no. 9, pp. 134-141, Sep. 2017.

[34] M. Rice, Digital Communications: A Discrete-Time Approach. Pearson Prentice Hall, 2009.

[35] J. Grotz, B. Ottersten, and J. Krause, "Data-aided frequency synchronization under interference limited conditions," in 2005 IEEE 61st Vehicular Technology Conference, vol. 4. IEEE, 2005, pp. 2683-2686.

[36] _ - "Joint channel synchronization under interference limited conditions," IEEE transactions on wireless communications, vol. 6 , no. 10 , pp. 3781-3789, 2007.

[37] A. Shah and B. Daneshrad, "A practical two-stage mmse based mimo detector for interference mitigation with non-cooperative interferers," EURASIP Journal on Wireless Communications and Networking, vol. 2011, no. 1, pp. 1-14, 2011.

[38] T. Su and H. Ling, "On modeling mutual coupling in antenna arrays using the coupling matrix," Microwave and Optical Technology Letters, vol. 28 , no. 4, pp. 231-237, 2001.

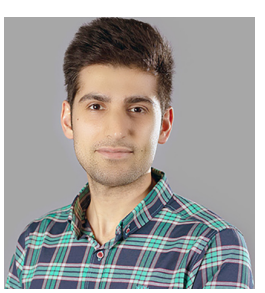

Masoud Abbasi Alaei was born in Babolsar, Iran, in 1989. He received the B.Sc. degree in electrical engineering from the University of Mazandaran, Iran, in 2011 and the M.Sc. degree in electrical engineering (Digital Hardware design) from Shahid Beheshti University, Iran, in 2014. He has worked for about three years as a freelancer FPGA designer and instructor in Iran. $\mathrm{He}$ is currently a Ph.D. student with the radio system group (RS), faculty of electrical engineering, computer science, and mathematics (EEMCS), University of Twente. His focus is on developing hybrid beamforming to mitigate interference in the MIMO communication system.

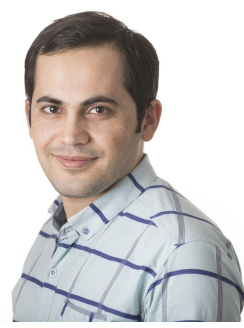

Sajad Golabighezelahmad (Student Member, IEEE) received the B.Sc. and M.Sc. degrees in electrical engineering from the K. N. Toosi University of Technology, Tehran, Iran, and Amirkabir University of Technology, Tehran, Iran, in 2011 and 2013, respectively. He is currently pursuing the Ph.D. degree with the IC Design Group, University of Twente, Enschede, The Netherlands.

His current research is focused on RF front-end design for multiple-input multiple-output (MIMO) and beamforming applications.

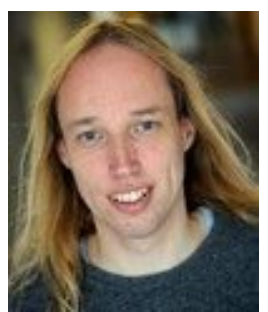

Pieter-Tjerk de Boer received the M.Sc. degree in applied physics and the Ph.D. degree in computer science from the University of Twente in 1996 and 2000, respectively, where he currently is an Associate Professor in the Design and Analysis of Communication Systems group.

His research interests include communication networks, their mathematical performance modeling and efficient simulation techniques, and softwaredefined radio. 


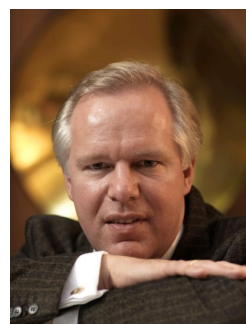

Frank van Vliet (M'96-SM'06) received the M.Sc. (Hons.) and Ph.D. degrees in electrical engineering (with a focus on MMIC filters) from the Delft University of Technology, Delft, The Netherlands, in 1992. He joined TNO, The Hague, The Netherlands (Netherlands Organization for Applied Scientific Research), in 1997, where he is currently a Principal Scientist responsible for MMIC, antenna, and transmit/receive module research. In 2007, he joined the Integrated Circuit Design Group, University of Twente, Enschede, The Netherlands, as a Professor in microwave integration, where he founded the Center for Array Technology. $\mathrm{He}$ has authored or coauthored over 150 peer-reviewed publications. His current research interests include MMICs in all their aspects, advanced measurement techniques, and phased-array technology. Dr. van Vliet is a member of the European Space Agencies (ESA) Component Technology Board (CTB) for microwave components, a member of the European Defence Agency (EDA) CapTech TCM, and chair of the 2012 European Microwave Integrated Circuit conference (EuMIC 2012). He founded the Doctoral School of Microwaves, and serves on the Technical Program Committee of EuMIC, the IEEE International Symposium on Phased Array Systems and Technology, the IEEE BiCMOS and Compound Semiconductor Integrated Circuits and Technology Symposium (IEEE BCICTS) and the IEEE Conference on Microwaves, Communications, Antennas and Electronic Systems (IEEE COMCAS). He was guest editor of the IEEE MTT 2013 Special Issue on Phased-Array Technology and served as the general chair of the European Microwave Week 2020.

His research interests include communication networks, their mathematical performance modeling and efficient simulation techniques, and softwaredefined radio.

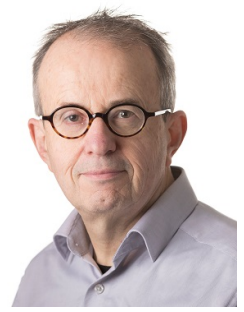

Eric A. M. Klumperink (Fellow, IEEE) was born on April 4th, 1960, in Lichtenvoorde, The Netherlands. He received the B.Sc. degree from HTS Enschede (1982), worked in industry on digital hardware and software, and then joined the University of Twente, Enschede, in 1984, shifting focus to analog CMOS circuit research. This resulted in several publications and his Ph.D. thesis "Transconductance Based CMOS Circuits: Circuit Generation, Classification and Analysis" (1997). In 1998, Eric started as Assistant Professor at the IC-Design Laboratory in Twente and shifted research focus to RF CMOS circuits (e.g. sabbatical at the Ruhr Universitaet in Bochum, Germany). Since 2006, he is an Associate Professor, teaching Analog \& RF IC Electronics and guiding PhD and MSc projects related to RF CMOS circuit design with focus on Software Defined Radio, Cognitive Radio and Beamforming. He served as an Associate Editor for the IEEE TCAS-II (2006-2007), IEEE TCAS-I (2008-2009) and the IEEE JSSC (2010-2014), as IEEE SSC Distinguished Lecturer (2014/2015), and as member of the technical program committees of ISSCC (2011-2016) and the IEEE RFIC Symposium (2011-..). He holds several patents, authored and co-authored 175+ internationally refereed journal and conference papers, and was recognized as 20+ ISSCC paper contributor over 1954-2013. He is a corecipient of the ISSCC 2002 and the ISSCC 2009 "Van Vessem Outstanding Paper Award".

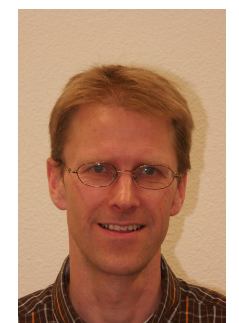

André B. J. Kokkeler has worked more than 6 years at Ericsson as a system engineer and 8 years at the Netherlands foundation for research in astronomy (ASTRON) as a scientific project manager. In 2003 he joined the University of Twente where he obtained his $\mathrm{PhD}$ degree in 2005. He currently is appointed as Associate Professor at the University of Twente.

$\mathrm{He}$ has a background in telecommunication, mixed signal design and signal processing architectures. Currently, his main interest lies in the area of the design of low-power architectures for telecommunications and computationally intensive applications. He is involved in research projects, sponsored by the Dutch and European governments and industry. His research focuses on efficient realization of digital signal processing for communications. 